\title{
Formation of Wide Binaries by Fragmentation
}

\author{
Peter Bodenheimer \\ UCO/Lick Observatory, Department of Astronomy and Astrophysics, \\ University of California, Santa Cruz, CA 95064, USA \\ Andreas Burkert \\ Max-Planck-Institut für Astronomie, Königstuhl 17, D-69117 \\ Heidelberg, Germany
}

\begin{abstract}
Although observations strongly suggest that fragmentation during the protostar collapse is the primary formation mechanism for wide binaries, the theoretical calculations as yet do not well explain the statistical properties of such systems. The results of a number of numerical simulations are discussed, and it is pointed out that, although fragmentation is obtained in such calculations, in many cases either the initial conditions are not realistic, or the calculations are insufficiently resolved, or the calculations have not been taken far enough to account for the accretion of most of the initial core material onto the components of the forming system. Certain aspects of the numerical results are, however, consistent with the fragmentation hypothesis.
\end{abstract}

\section{Introduction-Issues}

Fragmentation during the collapse of a rotating cloud has been generally accepted as the most likely process for the formation of wide binaries (Boss 1988). The earlier numerical simulations produced a wide variety of fragmentation outcomes. However, calculations with more realistic initial conditions and better numerical resolution indicate that the occurrence and understanding of fragmentation are more difficult than previously thought. A number of issues still remain to be resolved.

1. How are the general observed properties of binary and multiple systems explained? How can we understand the distribution of binaries according to period, the distribution of secondary masses for a given primary mass, and the distribution of orbital eccentricities?

2. How is the frequency of binary systems explained? How are single stars explained?

3. What are the details of the formation process? The possibilities for wide binaries include (1) spontaneous fragmentation during collapse of a rotating protostellar cloud; (2) induced fragmentation through cloud-cloud collisions; and (3) fragmentation, through one of the above processes, into 
a small cluster followed by interactions and captures. Is hierarchical fragmentation important?

4. What is the interaction between binaries and disks? How do orbits evolve after formation?

5. Why are close brown dwarf companions rare among G-K main-sequence stars, while wide brown dwarf companions and single brown dwarfs are apparently more frequent?

6. How does the cluster environment affect binary formation? Although most stars apparently form in clusters, most of the numerical simulations treat the collapse of individual molecular cloud cores.

7. What is the influence of magnetic fields?

8. How are binary formation processes modified in the early universe?

The observed properties of binaries are well described in other papers in this volume; however it should be noted here that it is clear that binary formation occurs along with star formation and that the general characteristics of binary systems have already become established by the time the stars reach the quasi-static pre-main-sequence phase (Mathieu 1994). The discovery of binary protostars is already underway (Mundy; this volume); for example, millimeter observations of L1551 NE (Moriarty-Schieven et al. 2000) show two components with unequal fluxes and a separation of about $300 \mathrm{AU}$, right in the range found by several numerical simulations. Also, $\mathrm{N}_{2} \mathrm{H}^{+}$observations of the Bok globule CB 230 (Launhardt, Sargent, \& Zinnecker 2000) show a binary of separation of about $4000 \mathrm{AU}$, on the high end of the angular momentum distribution for main-sequence systems (Duquennoy \& Mayor 1991).

\section{Numerical Calculations}

\subsection{Initial Conditions}

A wide variety of numerical simulations have been published over the past 20 years. We here state the typical assumed initial conditions for these simulations and compare them with observations, concentrating on the simulations that start with parameters appropriate to the dense cores of molecular clouds.

Cloud has an assumed geometry: spherical, or cylindrical, or oblate, or prolate. Observationally, cores appear to be non-spherical with perhaps a prolate shape with an axis ratio of 2:1.

Cloud has no infall motion and is rotating uniformly. In fact more than one-half of observed cores have a detectable gradient in the line-of-sight velocity, which is interpreted as a rotation; at a size of $0.1 \mathrm{pc}$, the typical angular velocity $\Omega \approx 4 \times 10^{-13} \mathrm{~s}^{-1}$ and specific angular momentum $J / M \approx 10^{21}$ $\mathrm{cm}^{2} \mathrm{~s}^{-1}$ (Goodman et al. 1993). 
Cloud has a mass of about 1 solar mass and a radius of 0.05 parsec, consistent with observations except that the typical core has a mass of a few solar masses.

Cloud has a distribution of density that is somewhat centrally condensed. Observationally, cores have a distribution of density near $\rho \propto R^{-2}$ in the outer regions, beyond a few thousand $\mathrm{AU}$, but closer to $\rho \propto R^{-1}$ in the inner regions (André, Ward-Thompson, \& Motte 1996; Ward-Thompson, Motte, \& André 1999).

Cloud has an initial assumed density perturbation, for example random noise or a $10 \% \mathrm{~m}=2$ mode. In fact, cloud density structure seems to be somewhat irregular. Core linewidths at a scale of $0.1 \mathrm{pc}$ are superthermal suggesting some turbulence (Barranco \& Goodman 1998), but the turbulent motions are subsonic.

Cloud is isothermal at $\mathrm{T} \approx 10 \mathrm{~K}$ and has a number density of $10^{4}$ to $10^{5} \mathrm{~cm}^{-3}$, in agreement with observations. These parameters indicate that the cores are near virial equilibrium.

The main parameters are:

$$
\begin{gathered}
\alpha=\text { thermal energy/|gravitational energy } \mid \\
\beta=\text { rotational energy/|gravitational energy } \mid \text {. }
\end{gathered}
$$

\subsection{Is there a Criterion for Fragmentation?}

For the case of cloud cores of uniform density and uniform rotation a number of analytical and numerical studies have been done. Analytic results showed (Tohline 1981; Hayashi et al. 1982) that collapsing rotating clouds fragment for $\alpha \beta<0.12$. However even the early numerical simulations, which were not well resolved, did not agree with the analytic result and indicated, with considerable uncertainty, that fragmentation occurred for $\alpha<0.4$, practically independent of $\beta$ (Bodenheimer, Tohline, \& Black 1980). Recent high-resolution collapse calculations with an SPH code that always resolves the Jeans mass (Tsuribe \& Inutsuka 1999a) also show that initially uniform clouds fragment only for $\alpha<0.4$ with a slight dependence on $\beta$; corresponding semi-analytical calculations give the same result (Tsuribe \& Inutsuka 1999b).

For the more realistic case of non-uniform density, the situation has not been explored very systematically. Boss (1993) showed that for somewhat prolate clouds with a Gaussian initial density profile, the critical value of $\alpha$ is reduced to about 0.3. Assuming a profile $\rho \propto R^{-1}$, Burkert, Bate, \& Bodenheimer (1997) showed that several fragments were produced near the centers of clouds with initial $\alpha=0.35$ and two different values of $\beta$. On the other hand, Tsai $\&$ Bertschinger (1989) showed that the singular isothermal sphere $\left(\rho \propto R^{-2}\right)$ is stable against fragmentation. However, the value of $\alpha$ for such a sphere is 0.5 . In general, as the degree of central concentration increases, the parameter space available for fragmentation seems to shrink (see also Tsuribe \& Inutsuka 2000). Given the large number of parameters needed to describe the initial condition for collapse, it seems unlikely that a simple criterion can be derived; 


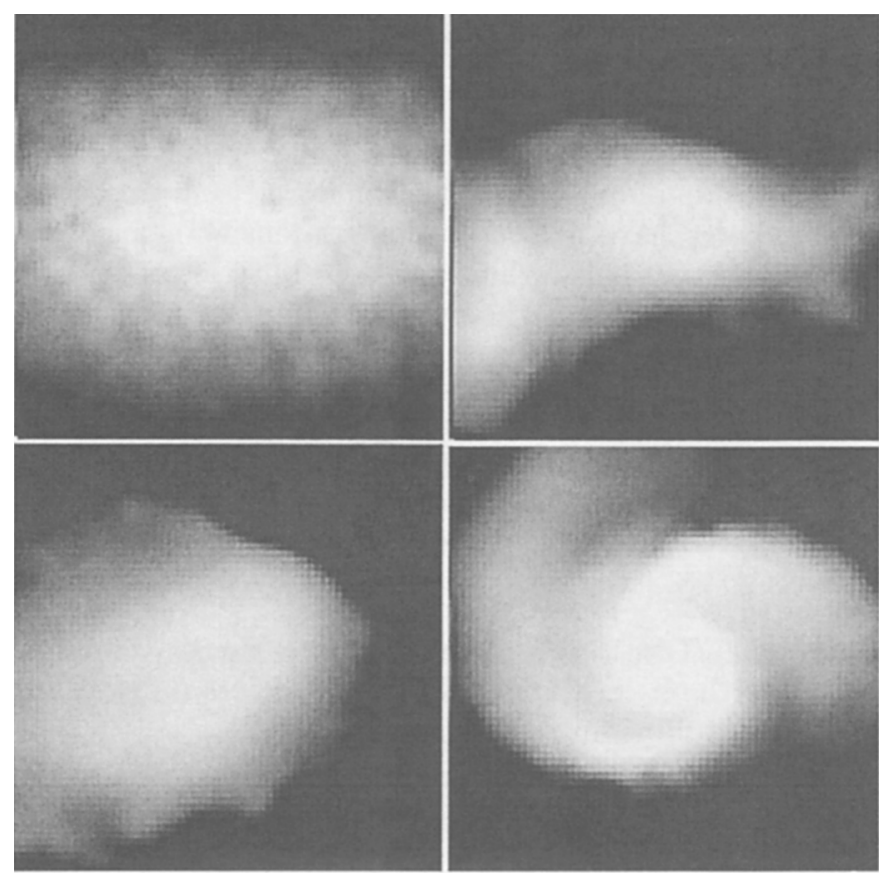

Figure 1. Four stages during the collapse of a prolate cloud core with $\alpha=0.47$ plus subsonic turbulence. The greyscale indicates the logarithm of the surface density, viewed toward the equatorial plane. Upper left: time $=8.7 \times 10^{10} \mathrm{~s}$, plot scale $=10^{17} \mathrm{~cm}, \log \rho_{\max }=$ -17.21. Upper right: time $=8.7 \times 10^{11} \mathrm{~s}$, plot scale $=5 \times 10^{16} \mathrm{~cm}, \log$ $\rho_{\max }=-16.6$. Lower left: time $=1.74 \times 10^{12} \mathrm{~s}$, plot scale $=5 \times 10^{16}$ $\mathrm{cm}, \log \rho_{\max }=-15.8$. Lower right: time $=2.3 \times 10^{12} \mathrm{~s}$, plot scale $=1 \times 10^{15} \mathrm{~cm}, \log \rho_{\max }=-7.6$.

for example the typical cloud core is somewhat turbulent and probably not uniformly rotating. However the approximate rule that the collapsing cloud fragments into a number of pieces equal to the number of Jeans masses at the initial state (Larson 1978) is roughly verified in a number of simulations that are not highly centrally condensed.

\subsection{Questions}

The above discussion suggests some more specific questions regarding the outcome of fragmentation calculations.

1. A typical molecular cloud core is centrally condensed and has $\alpha \approx 0.4$, $\beta \approx 0.02$. Will it fragment?

2. Numerical resolution is important and the Jeans condition must be satisfied (Bate \& Burkert 1997; Truelove et al. 1997, 1998). For highly resolved calculations, do different numerical codes give consistent results? 


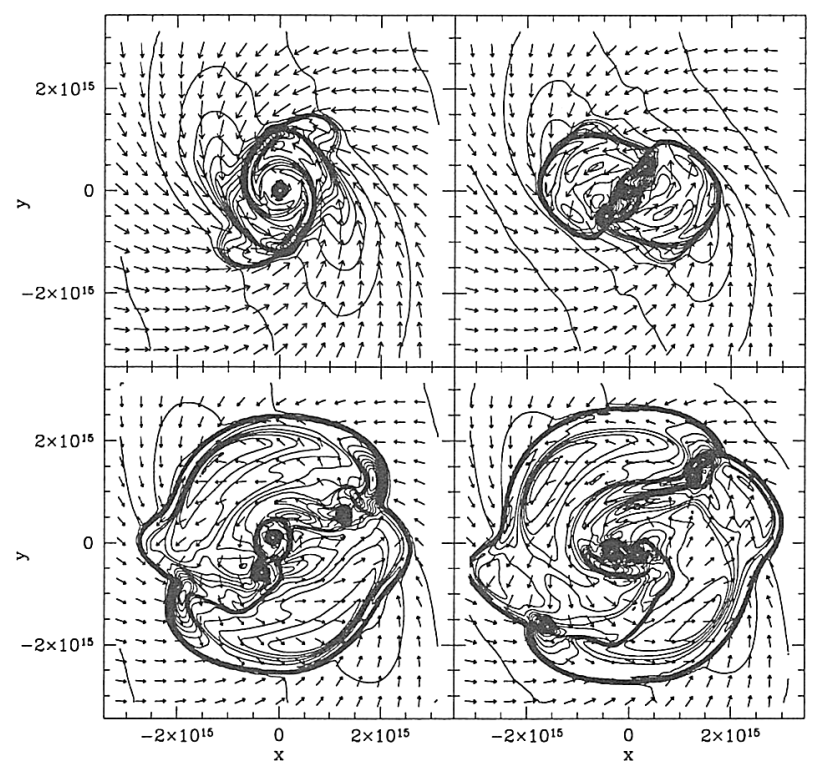

Figure 2. Contours of equal density and velocity vectors in the equatorial plane during the fragmentation of a cloud with initial $\alpha=0.35$ and $\beta=0.23$, as calculated with a nested-grid code (Burkert, Bate, \& Bodenheimer 1997). Upper left: $t=1.176 \times 10^{12} \mathrm{~s}$; upper right: $t=1.190 \times 10^{12} \mathrm{~s} ;$ lower left: $t=1.209 \times 10^{12} \mathrm{~s} ;$ lower right: $t=1.217 \times 10^{12} \mathrm{~s}$. Scale is in $\mathrm{cm}$.

3. Do fragmentation results depend strongly on the form and amplitude of the assumed initial perturbation?

4. Is fragmentation of a rotating collapsing cloud a reasonable solution to the problem of formation of a wide binary?

In the next section the general results of fragmentation calculations are discussed with a view toward answering these questions.

\section{Fragmentation: Results}

Fragmentation calculations have many possible outcomes, for example binary formation (Boss 1993; Bate, Bonnell, \& Price 1995), induced fragmentation by an initial central binary of further fragments in a surrounding disk (Bonnell \& Bate 1994), formation of a small cluster (Monaghan \& Lattanzio 1991; Boss 1996; Klapp \& Sigalotti 1998), fragmentation of filaments (Monaghan 1994; Inutsuka \& Miyama 1997), or formation of a binary plus low-mass fragments (Burkert \& Bodenheimer, unpublished).

The first question posed above has been addressed in a number of numerical simulations. For example Boss (1993) shows that for the appropriate values of $(\alpha, \beta)$ a prolate core does fragment if the axis ratio is $2: 1$ but does not fragment 


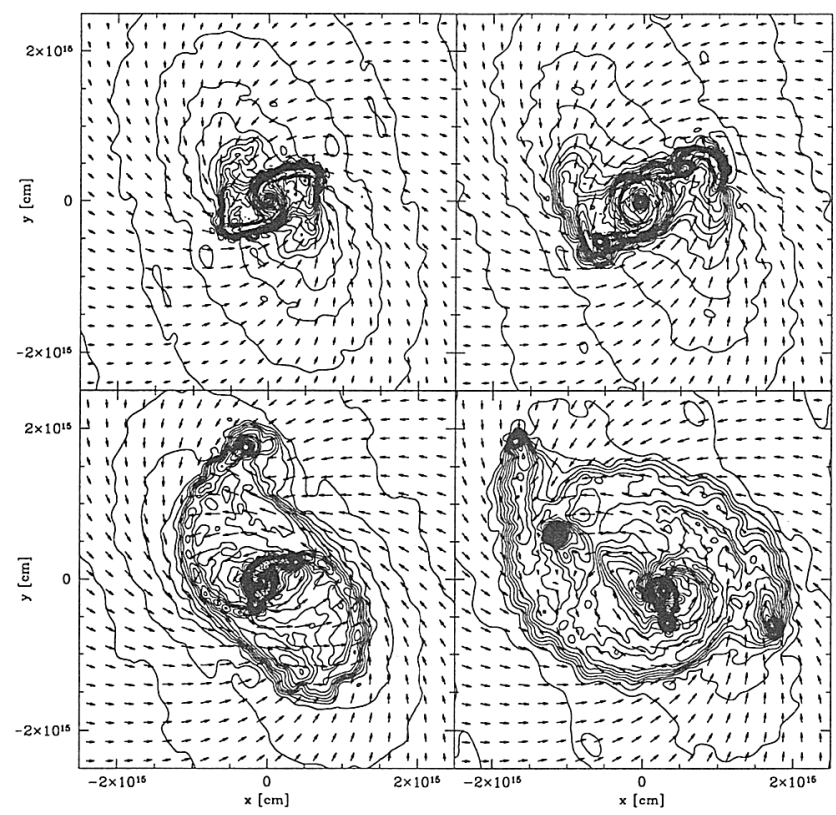

Figure 3. Contours of equal density and velocity vectors in the equatorial plane during the fragmentation of a cloud with initial $\alpha=0.35$ and $\beta=0.23$, as calculated with an SPH code (Burkert, Bate, \& Bodenheimer 1997). Upper left: $t=1.135 \times 10^{12} \mathrm{~s} ;$ upper right: $t=1.160 \times 10^{12} \mathrm{~s} ;$ lower left: $t=1.175 \times 10^{12} \mathrm{~s} ;$ lower right: $t=1.907 \times 10^{12} \mathrm{~s}$.

if the axis ratio is $1.5: 1$. The results of Tsuribe \& Inutsuka (2000) indicate that a centrally condensed spherical cloud with $\alpha=0.4$ would not fragment during the isothermal phase. Another example, which includes a moderate turbulence, is shown in Figure 1. The starting point is a cloud of $1 \mathrm{M}_{\odot}$ with a uniform sound speed of $1 \times 10^{4} \mathrm{~cm} \mathrm{~s}^{-1}$ with no ordered rotation and with a dimension $R_{\max }=1.088 \times 10^{17} \mathrm{~cm}$. The cloud is prolate with a density profile

$$
\rho(r)=\rho_{c} \exp \left(-\frac{x^{2}}{a^{2}}-\frac{y^{2}+z^{2}}{b^{2}}\right)
$$

where the central density $\rho_{c}=6.2 \times 10^{-18} \mathrm{~g} \mathrm{~cm}^{-3}, a=R_{\max } / 1.73$, and $b=a / 2$. A turbulent velocity field with $P(k) \sim k^{-4}$ is imposed, giving a ratio of kinetic energy to gravitational potential energy of 0.3 and a ratio of internal energy to gravitational potential energy of 0.47 . The subsonic turbulent velocity field results in a net specific angular momentum $j=3.1 \times 10^{20} \mathrm{~cm}^{2} \mathrm{~s}^{-1}$.

The isothermal evolution of the collapse is followed with an SPH code of 40,000 particles. The first frame, at an early time, shows evidence of a turbulent structure. In the second frame, two condensations appear, which later merge, but turbulence prevents overall collapse. In the third frame, a single core of almost uniform density has formed; it is about to collapse. The fourth frame shows, on a much smaller scale, a disk with a density of about $10^{-11} \mathrm{~g} \mathrm{~cm}^{-3}$, which is starting to heat, and a central object with much higher density. By the end of the simulation no fragmentation had occurred; further calculations 
are required. This calculation and others therefore indicate that the standard molecular cloud core is not likely to fragment during the early collapse phases, although the subsequent adiabatic evolution remains to be investigated. If the initial core has highly supersonic turbulence (Klein \& Fisher; this volume) fragmentation does occur; however for typical low-mass star-forming cores, such turbulence is not observed.

The typical fragmentation calculation, including the one shown in Figure 1, has not been carried out long enough so that most of the material in the original cloud has collapsed to the equatorial plane. Thus the final outcome in these systems, as influenced by fragmentation at later stages as well as by captures, mergers, and escapes, is not known. Under the assumption that an initial binary fragmentation does take place and there is no further fragmentation, Bate (2000) has followed approximately the accretion of the rest of the cloud material onto the binary. He finds that an initial state of large mass ratio tends to evolve towards smaller mass ratio, and that a system with low angular momentum is more likely to evolve to a system with equal masses than one with high angular momentum.

Turning to the second question, results may depend qualitatively on numerical resolution, particularly if the Jeans condition is not satisfied. Even if it is satisfied, calculations must show numerical convergence. A number of comparisons have been made between different numerical codes. For example, Burkert, Bate, \& Bodenheimer (1997) compared the collapse of centrally condensed clouds as calculated with a grid code and with an SPH code. The results are shown in Figures 2 and 3. Apart from showing that the two codes give qualitatively the same results, these figures also demonstrate that the formation of an initial central binary in a close orbit can induce the formation of additional fragments farther out, and that the fragmentation process can lead to a small cluster. Further tests are described in Bodenheimer et al. (2000) and Boss et al. (2000). We may conclude that different numerical codes with similar degree of resolution produce about the same results; however the question of numerical convergence has not been investigated thoroughly enough. The effect of the initial perturbation (question 3 above) has also not been investigated extensively, but existing studies, for example Tsuribe \& Inutsuka (1999a) and Bodenheimer, Yorke, \& Burkert (1999), indicate that the occurrence of fragmentation depends only slightly on the form of the initial perturbation, but the details of how the initial fragmentation phase proceeds is likely to be strongly affected.

\section{Conclusions}

In summary, we now turn to the fourth question posed above: Does fragmentation during a rotating collapse explain the properties of wide binaries? This theory has the following advantages:

1. A wide range of orbital angular momenta is predicted, depending on the angular momentum of the initial cloud core plus effects of captures after fragmentation into a small cluster.

2. Calculations do indicate presence of circumstellar and circumbinary disks (see, for example, Bodenheimer et al. 2000). 
3. This process predicts that components of young binaries are coeval, in agreement with most comparisons of young binaries with pre-main sequence evolutionary tracks. However, this constraint is not very strong, since derived ages are not accurate to better than 1-2 Myr.

4. The calculations predict eccentric orbits, in agreement with the fact that most binary orbits, except the tidally circularized very close orbits, have a wide range of eccentricities.

5. Cloud core angular momenta are of the correct order of magnitude to explain wide binary orbits. Although Simon (1992) points out that core angular momenta are a factor 10 larger than the typical orbital angular momenta of wide binaries, in fact the typical core angular momenta are probably overestimated by up to a factor of 3 (Burkert \& Bodenheimer 2000). Furthermore, not all of the core angular momentum ends up in the binary; according to one of the very few calculations that has been carried to the point where accretion onto a forming binary is essentially complete, the result shows that roughly half of the core angular momentum does not end up in the binary orbit but is deposited in a circumbinary disk (Bate et al. 1995).

6. The calculated fragments are themselves unstable to collapse and probably to fragmentation, so hierarchical multiples could be explained.

However, the fragmentation hypothesis, and in particular the numerical calculations which support it, also have a number of problems:

1. For standard core parameters, fragmentation tends to set in just at the density where the center of the cloud is beginning to heat. Many calculations have not treated radiation transport but have assumed adiabatic heating starting from an assumed critical density. The precise treatment of radiation transfer may be important.

2. The role of magnetic fields in regulating the protostellar collapse and fragmentation phase has not been explored in sufficient detail.

3. Since there are so many possible initial conditions, the statistics of mass ratio distributions and eccentricities will be difficult to derive from detailed three-dimensional calculations.

4. Numerical simulations have not been well compared with observations, for example, with millimeter fluxes.

5. It is much more difficult to explain close binaries by this process, but it is highly questionable that there are two completely independent processes for binary formation, since the transition in observables from close binaries to wide binaries is so smooth. 


\section{References}

André, P., Ward-Thompson, D., \& Motte, F. 1996, A\&A, 314, 625

Barranco, J. A., \& Goodman, A. A. 1998, ApJ, 504, 207

Bate, M. R. 2000, MNRAS, 314, 33

Bate, M. R., Bonnell, I., \& Price, N. 1995, MNRAS, 277, 362

Bate, M. R., \& Burkert, A. 1997, MNRAS, 288, 1060

Bodenheimer, P., Burkert, A., Klein, R. I., \& Boss, A. P. 2000, in Protostars and Planets IV, ed. V. Mannings, A. P. Boss \& S. Russell (Tucson: Univ. of Arizona Press), 675

Bodenheimer, P., Tohline, J. E., \& Black, D. C. 1980, ApJ, 242, 209

Bodenheimer, P., Yorke, H. W., \& Burkert, A. 1999, in Numerical Astrophysics, ed. S. M. Miyama, K. Tomisaka \& T. Hanawa (Dordrecht: Kluwer), 123

Bonnell, I., \& Bate, M. R. 1994, MNRAS, 269, L45

Boss, A. P. 1988, Comment. Astrophys., 12, 169

Boss, A. P. 1993, ApJ, 410, 157

Boss, A. P. 1996, ApJ, 468, 231

Boss, A. P., Fisher, R. T., Klein, R. I., \& McKee, C. F. 2000, ApJ, 528, 325

Burkert, A., Bate, M. R., \& Bodenheimer, P. 1997, MNRAS, 289, 497

Burkert, A., \& Bodenheimer, P. 2000, ApJ, in press

Duquennoy, A., \& Mayor, M. 1991, A\&A, 248, 485

Goodman, A. A., Benson, P., Fuller, G., \& Myers, P. 1993, ApJ, 406, 528

Hayashi, C., Narita, S., \& Miyama, S. M. 1982, Prog. Theor. Phys., 68, 1949

Inutsuka, S., \& Miyama, S. M. 1997, ApJ, 480, 681

Klapp, J., \& Sigalotti, L. DiG. 1998, ApJ, 504, 158

Larson, R. B. 1978, MNRAS, 184, 69

Launhardt, R., Sargent, A. I., \& Zinnecker, H. 2000, in Science with the Atacama Large Millimeter Array, ASP Conference Series, ed. A. Wootten (San Francisco: Astronomical Society of the Pacific), 103

Mathieu, R. 1994, ARA\&A, 32, 465

Monaghan, J. J. 1994, ApJ, 420, 692

Monaghan, J. J., \& Lattanzio, J. C. 1991, ApJ, 375, 177

Moriarty-Schieven, G. H., Powers, J. A., Butner, H. M., Wannier, P. G., \& Keene, J. 2000, preprint

Simon, M. 1992, in ASP Conf. Ser. 32, Complementary Approaches to Double and Multiple Star Research, ed. H. A. McAlister \& W. I. Hartkopf (San Francisco: ASP), 41

Tohline, J. E. 1981, ApJ, 248, 717

Truelove, J. K., Klein, R. I., McKee, C. F., Holliman, J. H., II, Howell, L. H., \& Greenough, J. A. 1997, ApJ, 489, L179

Truelove, J. K., Klein, R. I., McKee, C. F., Holliman, J. H., II, Howell, L. H., Greenough, J. A., \& Woods, D. T. 1998, ApJ, 495, 821

Tsai, J., \& Bertschinger, E. 1989, Bull. A. A. S., 21, 1089 
Tsuribe, T., \& Inutsuka, S. 1999a, ApJ, 523, L155

Tsuribe, T., \& Inutsuka, S. 1999b, ApJ, 526, 307

Tsuribe, T., \& Inutsuka, S. 2000, in Poster Proceedings of IAU Symposium No. 200 on The Formation of Binary Stars, ed. B. Reipurth \& H. Zinnecker, 184

Ward-Thompson, D., Motte, F., \& André, P. 1999, MNRAS, 305, 143
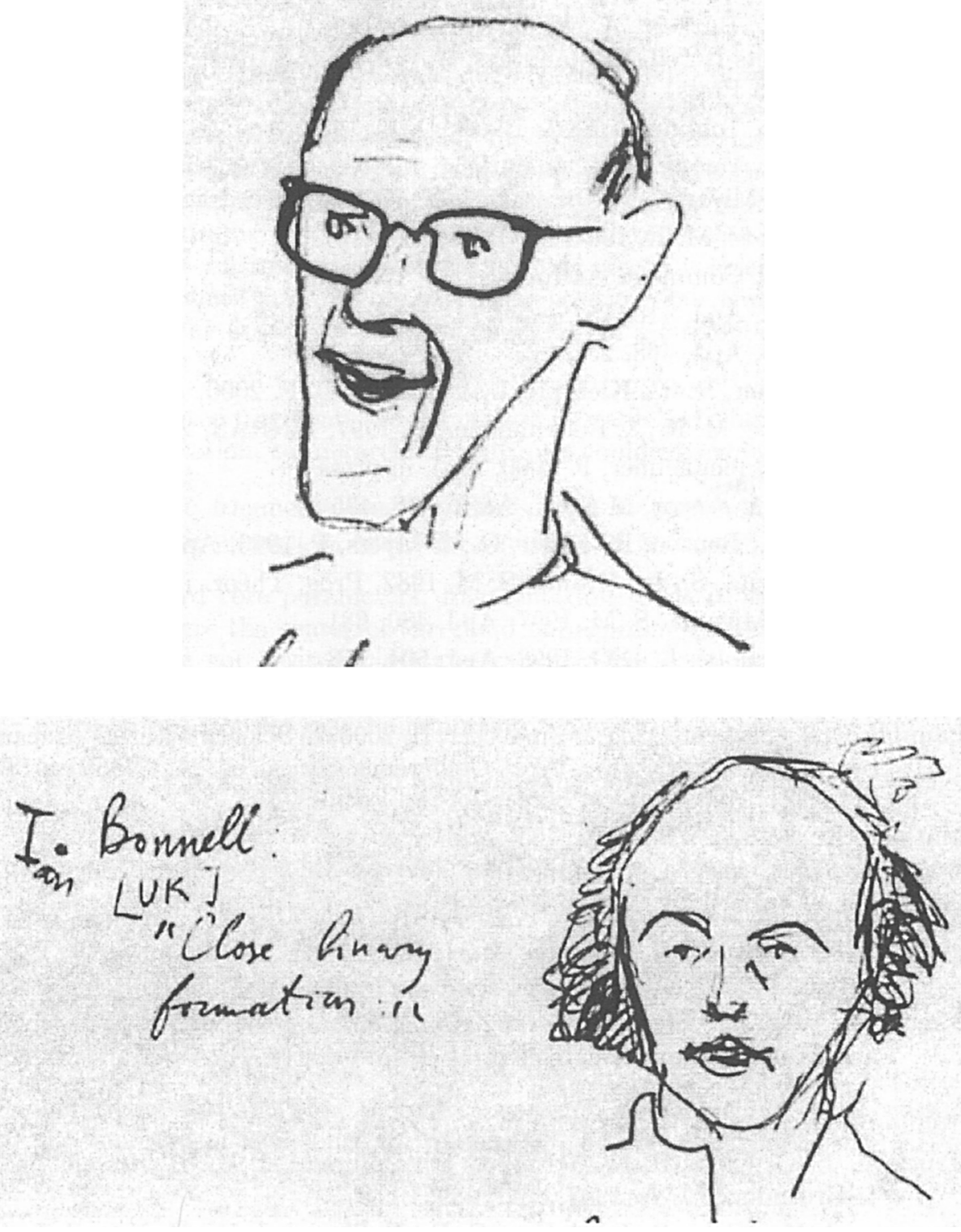

Peter Bodenheimer and Ian Bonnell, sketched by Y. Balega 